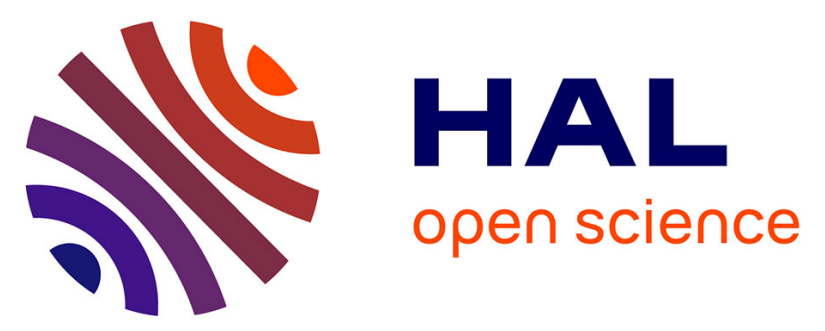

\title{
Central and storage carbon metabolism of the brown alga Ectocarpus siliculosus: insights into the origin and evolution of storage carbohydrates in Eukaryotes
}

Gurvan Michel, Thierry Tonon, Delphine Scornet, J. Mark Cock, Bernard Kloareg

\section{To cite this version:}

Gurvan Michel, Thierry Tonon, Delphine Scornet, J. Mark Cock, Bernard Kloareg. Central and storage carbon metabolism of the brown alga Ectocarpus siliculosus: insights into the origin and evolution of storage carbohydrates in Eukaryotes. New Phytologist, 2010, 188 (1), pp.67-81. 10.1111/j.14698137.2010.03345.x . hal-01806417

\section{HAL Id: hal-01806417 https://hal.science/hal-01806417}

Submitted on 16 Nov 2018

HAL is a multi-disciplinary open access archive for the deposit and dissemination of scientific research documents, whether they are published or not. The documents may come from teaching and research institutions in France or abroad, or from public or private research centers.
L'archive ouverte pluridisciplinaire $\mathbf{H A L}$, est destinée au dépôt et à la diffusion de documents scientifiques de niveau recherche, publiés ou non, émanant des établissements d'enseignement et de recherche français ou étrangers, des laboratoires publics ou privés. 
1 The carbohydrate metabolism of the brown alga Ectocarpus siliculosus.

2 Part I: Central and storage carbon metabolism. Insights into the origin and

3

4

5

6

7

8

9

10

11 Roscoff, Bretagne, France

12 *Corresponding author: Tel (33) 2982923 30, Fax (33) 2982923 24, E-mail: gurvan@sb-

$13 \underline{\text { roscoff.fr }}$

14

15 Summary: 196 words

16 Total word count: 6365 words

17 Introduction: 715 words

18 Materials and Methods: 252 words

19 Results: 2371 words

20 Discussion: 3027 words

21 Tables: 0

22 Figures: 6

23 Supporting Information Tables : 3

Bernard KLOAREG ${ }^{1,2}$

${ }^{1}$ UPMC University Paris 06, UMR 7139 Marine Plants and Biomolecules, Station Biologique de Roscoff, F-29682 Roscoff, Bretagne, France

${ }^{2}$ CNRS, UMR 7139 Marine Plants and Biomolecules, Station Biologique de Roscoff, F-29682

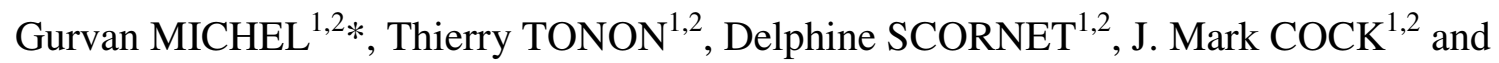
Bemard KLOAREG 


\section{Summary}

2 - Brown algae exhibit a unique carbon storage metabolism. The photoassimilate D-fructose

3 6-phosphate is not used to produce sucrose but is converted into D-mannitol. These

4 seaweeds also store carbon as $\beta$-1,3-glucan (laminarin), thus markedly departing from

5 most living organisms, which use $\alpha$-1,4-glucans (glycogen or starch).

- We identify the enzymes involved in carbon storage in the genome of the brown alga Ectocarpus siliculosus and trace their evolutionary origins. Ectocarpus possesses a complete set of enzymes for synthesis of mannitol, laminarin and trehalose, while the pathways for sucrose, starch and glycogen are completely absent.

- The synthesis of $\beta$-1,3-glucans appears to be a very ancient eukaryotic pathway. Brown

11 algae inherited the trehalose pathway from the red algal progenitor of phaeoplasts, while the mannitol pathway was acquired by lateral gene transfer from Actinobacteria. The starch metabolism of the red algal endosymbiont was entirely lost in the ancestor of

14 Stramenopiles, as was glycogen metabolism from the ancestral host.

- In the light of these novel findings we question the validity of the "Chromalveolate hypothesis". We also propose that $\beta$-1,3-glucans were the archetypal carbon storage compound in Eukaryotes. In most extant lineages they were supplanted by glycogen following mitochondrial endosymbiosis.

20 Keywords: brown algae, Chromalveolate, Eukaryotic evolution, beta-1,3-glucan, glycogen, 21 starch, mannitol, trehalose 


\section{Introduction}

Brown algae (Phaeophyceae) are photosynthetic, multicellular organisms that dominate

3 rocky coastal environments. These macroalgae are members of the Stramenopiles, a

4 eukaryotic phylum which also includes diatoms, Oomycetes and various protists (Fig. 1) (Baldauf, 2008). Stramenopiles are characterized by the occurrence in their life history of cells with two unequal flagella and the monophyly of this group has been confirmed by molecular phylogenies (Ben Ali et al., 2001). Stramenopile plastids arose via a secondary endosymbiotic event, in which a unicellular red alga was engulfed by an ancestral protist (Reyes-Prieto et al., 2007). Other eukaryotic lineages also possess secondary plastids derived from red algae, particularly the Alveolates, Haptophytes and Cryptophytes (Fig. 1). CavalierSmith proposed that a single secondary endosymbiosis with a red alga gave rise to the plastid ancestor of all these eukaryotic groups (the Chromalveolate hypothesis) and, therefore, that the host lineages form a monophyletic super-group designated as Chromalveolates (CavalierSmith, 1999). The Chromalveolate hypothesis has been intensely debated in the last decade and is still a highly contentious issue (Bodyl et al., 2009; Keeling, 2009; Lane \& Archibald, 2009).

This complex evolutionary history of brown algae is reflected by the uniqueness of their carbohydrate metabolism. With respect to the outflow of the Calvin cycle the photoassimilate D-fructose-6-phosphate (F6P) is not used by brown algae to produce sucrose as in higher plants, but it is mainly converted into D-mannitol. This alcohol sugar is localized in the cytosol and is by far the most abundant, organic soluble compound in brown algae (Gravot et al., 2010, this issue). Mannitol is synthesized in two steps: F6P is reduced by mannitol-1phosphate 5-dehydrogenase (M1PDH) into mannitol-1P, which is then converted into mannitol by mannitol-1-phosphatase (M1Pase). These enzymatic activities have been measured in several brown algae (Ikawa et al., 1972), but the corresponding genes have not 
1 been described yet. Mannitol is thought to be recycled by mannitol-2-dehydrogenase (M2DH)

2 and hexokinase (Iwamoto \& Shiraiwa, 2005).

3 Brown algae and other Stramenopiles also possess unique carbon storage polysaccharides.

4 Most living organisms store carbon as linear or branched $\alpha$-1,4-glucans: glycogen in 5 Opisthokonta (animals and fungi) and in most bacteria, or starch in the diazotrophic 6 cyanobacteria and Archaeplastida (i.e. red algae, green algae and plants, Deschamps et al., 7 2008). In contrast, the storage polysaccharide of brown algae is laminarin, a vacuolar $\beta-1,3-$ 8 glucan with occasional $\beta$-1,6-linked branches (Percival \& Ross, 1951). This polysaccharide is 9 polydisperse, consisting of a minor G-series with polymers containing only glucose residues, and a more abundant M-series with glucans terminated with a 1-linked D-mannitol residue

11 (Read et al., 1996). Diatoms and oomycetes also produce vacuolar $\beta$-1,3-glucans, known as chrysolaminarin (Beattie et al., 1961) and mycolaminarin (Wang \& Bartnicki-Garcia, 1974), respectively. These storage polysaccharides have a branched structure similar to that of laminarin, but they do not contain mannitol residues. In the sieve elements of Laminariales, plates are made of microfibrillar $\beta$-1,3-glucans deposits, reminiscent of plant callose (Parker \& Huber, 1965). Self-assembling linear $\beta$-1,3-glucans also occur as structural components in the cell wall of Oomycetes (Bartnicki-Garcia, 1968).

Experiments with radioactive carbon demonstrated that laminarin and mannitol are interchangeable storage compounds in brown algae, as are sucrose and starch in higher plants 20 (Yamaguchi et al., 1966). In kelps mannitol can be remobilized and translocated via the sieve tubes from mature tissues to supply the rapidly-growing parts of the alga with carbon (Schmitz \& Lobban, 1976; Lobban \& Harrison, 1994).

The molecular bases of carbohydrate metabolism in brown algae are essentially 24 uncharacterized. Until recently, expressed sequence tag (EST) libraries were the only molecular data available for inferences on central and storage carbon metabolisms in brown 
1 algae. Analysis of a cDNA library produced from sporophytes of Laminaria digitata retrieved

2 six partial open reading frames corresponding to genes potentially involved in central carbon 3 metabolism (Moulin et al., 1999). The complete genome sequence of the brown alga 4 Ectocarpus siliculosus, from the order Ectocarpales (Charrier et al., 2008), is now available 5 (Cock et al., 2010), providing, for the first time, a comprehensive view of brown algal 6 carbohydrate metabolism.

7 Here we decipher the metabolic routes for central sugar metabolism and carbon storage in 8 brown algae and reconstruct the phylogenetic relationships of key enzymes in these metabolic 9 pathways. This analysis provides novel insights into the origin and evolution of carbohydrates 10 in Eukaryotes and the results raise doubts with regard to the Chromalveolate hypothesis. 


\section{Materials and methods}

\section{Identification and bioinformatic analyses of carbohydrate-related proteins}

3 The proteins involved in carbohydrate metabolism encoded by the E. siliculosus genome

4 were identified by homology with biochemically characterized proteins selected in the CAZY

5 (http://www.cazy.org/, Cantarel et al., 2009) and UniProt databases. For each identified

6 Ectocarpus protein, evidence of conserved protein modules was queried using the Pfam

7 database (Bateman et al., 2004). The presence of additional, orphan modules was detected by

8 BlastP searches against the UniProt database. Signal peptides and transmembrane helices

9 were predicted using HECTAR (Gschloessl et al., 2008) and TMHMM (Krogh et al., 2001), respectively. Numerous proteins involved in carbohydrate metabolism belong to families

11 encompassing several enzymatic activities and/or substrate specificities. To clarify their

12 function, these proteins were further analyzed by a phylogenetic approach. For each different

13 activity of a poly-specific family, a set of experimentally characterized proteins was selected

14 in the UniProt database. These representative proteins were aligned with their homologues from E. siliculosus using MAFFT with the iterative refinement method and the scoring matrix Blosum62 (Katoh et al., 2002). Phylogenetic trees were derived from this refined alignment

17 using the Maximum Likelihood method with the program PhyML (Guindon \& Gascuel, 2003). The reliability of the trees was always tested by bootstrap analysis using 100 resamplings of the dataset. The trees were displayed with MEGA (Kumar et al., 2004). The functional annotation of Ectocarpus proteins was based on the proximity to specific characterized proteins in the phylogenetic trees. Genomic comparisons were performed using the genomic BLAST server at NCBI (http://www.ncbi.nlm.nih.gov/sutils/genom_table.cgi). 


\section{Results}

2 From carbon fixation to carbohydrate biosynthesis: an overview of the carbohydrate 3 active enzymes in Ectocarpus siliculosus

4

As in most photosynthetic organisms, Rubisco is responsible for carbon fixation in brown algae, releasing two molecules of glycerate-3-phosphate (Assali et al., 1991). The enzymes converting this triose-phosphate into F6P are well conserved in E. siliculosus (Supporting Information Table S1). Based on bioinformatic predictions of signal peptides (Gschloessl et al., 2008), the corresponding isoenzymes mainly differ in their localization (cytosol, mitochondrial or plastid matrices). Interestingly, the gene Esi0187_0027 encodes a modular protein encompassing a triose-phosphate isomerase (TPI) fused to a glyceraldehyde-3phosphate dehydrogenase (GAPDH). Homologues of this translational fusion are only found in diatoms (Armbrust et al., 2004; Bowler et al., 2008) and Oomycetes (Tyler et al., 2006; Haas et al., 2009), suggesting that this bifunctional enzyme is characteristic of Stramenopiles. The TPI/GAPDH fusion-protein from the diatom Phaeodactylum tricornutum has been shown to be active and is localized in mitochondria (Liaud et al., 2000). The enzymes that equilibrate the hexose-phosphate pool (F6P, D-glucose-6P and D-glucose-1P) are also highly conserved, with two isoforms each of glucose-6 isomerase (GPI, Esi0060_0128 and Esi0266_0033) and of phosphoglucomutase (PGM, Esi0002_0317 and Esi0430_0005). Moreover, Esi0430_0005 displays an additional UDP-glucose-pyrophosphorylase (UGP) module at the N-terminus. Again, homologues of this UGP/PGM fusion-protein are only found in the genomes of Oomycetes and of the diatom $P$. tricornutum. In Thalassiosira pseudonana, these activities are however encoded by two distinct genes (Armbrust et al., 2004). The bifunctional enzymes TPI/GAPDH and UGP/PGM catalyze consecutive reactions, suggesting that these fusion events may have increased the efficiency of the metabolic steps leading to the activated form of glucose (UDP-glucose) in most Stramenopiles. 
1 Synthesis of polysaccharides and glycoconjugates is catalyzed by glycosyltransferases 2 (GT), which use activated sugar donors, or by transglycosylases. The cleavage of glycosidic 3 linkages is performed by glycoside hydrolases (GH) or by polysaccharide lyases (PL). 4 Carbohydrate esterases (CE) remove methyl or acetyl groups from substituted 5 polysaccharides. Collectively these enzymes are termed Carbohydrate Active enZYmes 6 (CAZYmes). Based on sequence similarities (Henrissat, 1991), CAZYmes have been 7 classified into more than 200 protein families (http://www.cazy.org/, Cantarel et al., 2009). 8 The genome of Ectocarpus encodes $41 \mathrm{GH}$ and 88 GT, but surprisingly lacks genes 9 homologous to known PL or CE (Supporting Information Table S2). This seaweed possesses 10 a slightly greater number of GH/GT genes than the marine green microalgae Micromonas and 11 Ostreococcus (Worden et al., 2009), but at least six times less than terrestrial plants. 12 Arabidopsis thaliana, for example, has 730 GH/GT genes (Henrissat et al., 2001). However, looking at the number of CAZY families, the difference between brown algae and land plants is less pronounced: Ectocarpus has members of $18 \mathrm{GH}$ and $32 \mathrm{GT}$ families, while Arabidopsis contains members of $34 \mathrm{GH}$ and $40 \mathrm{GT}$ families. The impressive number of CAZymes in plants is mainly explained by the presence of large multigenic families. For instance, Arabidopsis has 67 polygalacturonases (GH28), which participate in pectin recycling, and 121 GT1 that are mainly involved in secondary metabolite biosynthesis (Henrissat et al., 2001). In contrast, Ectocarpus features less functional redundancy, with fewer genes in each CAZY family. Interestingly, this seaweed contains some families which are absent from plants but are conserved with other phyla, such as bacteria (GH88, $\Delta-4,5$ unsaturated $\beta$-glucuronyl hydrolase), fungi (GT15, biosynthesis of cell wall mannoproteins), 23 animals and fungi (GH30, $\beta$-glucosidase; GT23, GT49 and GT54, N-glycosylation) and 24 Amoebozoa (GT60 and GT74, O-glycosylation of Skp1 subunits of E3 ubiquitin-protein 25 ligase). 


\section{Mannitol}

2 Three Ectocarpus proteins (Esi0017_0062, Esi0020_0181 and Esi0080_0017) share

3 significant sequence identity $(\sim 30 \%)$ with the M1PDH from the Apicomplexa Eimeria tenella. The only known M1Pase gene was also cloned from this parasite (Liberator et al., 1998), but no homolog has been found in Ectocarpus. Recycling of mannitol is probably performed by Esi0135_0010, which is similar to bacterial and fungal M2DH. However, no ortholog of eukaryotic hexokinases was found in E. siliculosus. Instead, this seaweed possesses a gene, Esi0139_0025, which is closely related to cyanobacterial fructokinases. Thus, brown algae apparently are an exception to the trend that broad specificity hexokinases are typical of multicellular eukaryotes, whereas sugar-specific kinases are distinctive of 11 bacteria and unicellular eukaryotes (Cardenas et al., 1998).

M1PDH genes have been identified in cDNA libraries from the brown algae L. digitata (Roeder et al., 2005) and Sargassum binderi (Wong et al., 2007), but are absent from the genome of diatoms and Oomycetes. Homologues of M1PDH have been found in only a few organisms so far, namely Gram positive bacteria, some fungi and two species of Micromonas

16 (Worden et al., 2009). The presence of M1PDH in Micromonas is surprising, since this gene is absent from the genomes of two other green algae, namely Ostreococcus (Derelle et al., 2006) and Chlamydomonas (Merchant et al., 2007), from the red alga Cyanidioschyzon merolae (Matsuzaki et al., 2004) and from terrestrial plants. However, mannitol has been reported in some other Prasinophytes (Kremer, 1980) and M1PDH activity was detected in

21 the green alga Platymonas subcordiformis (Richter \& Kirst, 1987). Phylogenetic analyses suggest that bacterial M1PDH genes were acquired independently by fungi and brown algae (Fig. 2a). In contrast, the M1PDH sequences of E. tenella and of the Micromonas species are nested within the brown algal clade. The Micromonas M1PDH genes (MICPUN_62892 and MICPUC_48208) both encode fusion proteins that include a haloacid dehalogenase-like 
1 domain (HAD). Interestingly the HAD superfamily contains various phosphatases, including

2 sucrose-phosphate and trehalose-phosphate phosphatases (Ridder \& Dijkstra, 1999). The two

3 Micromonas species possess a second HAD-like enzyme existing as a standalone protein

4 (MICPUC_47598 and MICPUN_101665). These M1PDH/HAD proteins are reminiscent of

5 the fusion sucrose-phosphate synthase / sucrose-phosphate phosphatases (SPS/SPP) and

6 trehalose-phosphate synthase / trehalose-phosphate phosphatases (TPS/TPP), suggesting that

7 the HAD modules provide the missing M1Pase activity. This assumption is further

8 strengthened by the identification in Ectocarpus of two standalone proteins (Esi0080_0016

9 and Esi0100_0020) highly similar to the Micromonas putative M1Pases (63\% sequence

10 identity with MICPUC_47598). In addition the putative M1Pase Esi0080_0016 is located

11 next to the M1PDH gene Esi0080_0017. This would be a rare example of functional

12 clustering in Ectocarpus (Cock et al., 2010). A conserved putative M1PAse was also found in

13 the EST library of the brown alga Fucus serratus (Pearson et al., 2009), while additional

14 significant homologues occur in some bacteria and fungi only. Comparison with crystal

15 structures of HAD-like phosphatases confirm that the catalytic machinery (Ridder \& Dijkstra,

16 1999) is strictly conserved in the putative M1Pases (data not shown). Phylogenetic analyses

17 indicate that brown algal putative M1Pases are also basal to the Micromonas standalone

18 enzymes, while putative MP1ases fused to M1PDH form a sister group (Fig. 2b).

\section{Non-reducing disaccharides}

20 Sucrose and trehalose are the most commonly occurring non-reducing disaccharides. Their

21 metabolic pathways are biochemically similar, but involve enzymes which are not

22 homologous (Paul et al., 2008). Phylogenetic analyses of sucrose-related proteins indicate that

23 plant sucrose metabolism was acquired from the cyanobacterial progenitor of chloroplasts

24 (Salerno \& Curatti, 2003). Sucrose metabolism is completely absent in Ectocarpus, as

25 deduced from the lack of genes encoding SPS (family GT4), sucrose synthase (GT4), SPP and 
1 invertases (families GH32 and GH100). These enzymes are also absent in diatoms and

2 Oomycetes, with the exception of the GH32 family of invertases present in Phytophthora

3 species. However, these latter enzymes are related to fungal orthologues, suggesting that

4 Phytophthora uses invertases to feed on plant sucrose. In contrast, Ectocarpus possesses a

5 complete trehalose pathway. This sugar is synthesized by a family of six bifunctional

6 enzymes, including a TPS (family GT20) fused to a TPP, while the recycling of trehalose is

7 assured by a single trehalase (family GH37).

8 TPS are widespread in bacteria, suggesting a prokaryotic origin for these

9 glycosyltransferases. Bacterial TPS exists in two forms, either as standalone protein or as a

10 fusion with TPP. Both types of TPS exist in fungi and they form two distinct clades in

11 phylogenetic trees (data not shown). In contrast, TPS are only present as fusion-proteins in

12 Dictyostelium discoideum (Amoebozoa), insects, red algae, green algae, plants, Alveolates and Stramenopiles. Phylogenetic analyses of the TPS and TTP domains of these fusion-

14 proteins result in similar topologies, with similar clades observed for both domains (Fig. 3),

15 even though the TPS domains are more divergent and score weaker bootstrap values.

16 Therefore the TPS and TPP domains have co-evolved, indicating that all these eukaryotic

17 phyla have acquired TPS directly as fusion-proteins. Bacterial and archaeal TPS domains constitute a well-supported clade, which was chosen as the outgroup (Fig. 3a). The TPS from insects emerge as an isolated cluster, while the fungal bifunctional TPS are distantly related to the TPS domain of D. discoideum. The TPS from Archaeplastida, Alveolates and

21 Stramenopiles form three distinct clades, which group together. The red alga C. merolae 22 possesses three TPS isoforms (CMI293C, CMO053C and CMP219C). Green algal and plant 23 TPS cluster together into two well-supported clades, which are sister groups of CMI293C and 24 CMO053C, respectively. However, CMP219C-like TPS genes are absent from the green 25 lineages (Fig. 3a), indicating either gene loss in the ancestor of green algae and plants or a 
1 specific gene duplication in red algae. Red algal TPS are basal to the TPS from Stramenopiles

2 and Alveolates, indicating that these eukaryotic phyla acquired TPS from ancestral red algal

3 endosymbiont(s). Ectocarpus possesses the three types of red algal TPS and has even evolved

4 a complex CMI293-like TPS family. Oomycetes conserved the CMP219C- and CMO053C-

5 like TPS but they lost the CMI293C-like TPS. In contrast, diatoms and Apicomplexa have

6 conserved only this latter type of TPS (Fig. 3a).

\section{$7 \quad$ Laminarin and other $\beta$-1,3-glucans}

The biosynthetic pathway of laminarin is essentially unknown, but we identified several genes which are likely to be involved in this metabolism in the Ectocarpus genome. This seaweed contains two cytosolic isoforms of UDP-glucose pyrophosphorylase (UGP, Esi0144_0004 and Esi0430_0005), supporting the assumption that UDP-glucose is the activated sugar needed for the production of laminarin. Beta-1,3-glucan synthases fall into two different GT families: the GT2, a polyspecific family which includes bacterial $\beta-1,3-$

14 glucan synthases, and the GT48, which only contains eukaryotic $\beta$-1,3-glucan synthases (Cantarel et al., 2009). Ectocarpus harbors eleven GT2, none of which are related to bacterial 3-1,3-glucan synthases (Michel et al., 2010, this issue). In contrast, the three members of the GT48 family (Esi0033_0138, Esi0193_0029 and Esi0338_0032) display significant similarities with plant callose synthases ( $\sim 35 \%$ sequence identity). The phylogenetic tree of the GT48 family (Fig. 4) is congruent with the currently accepted phylogeny of the Eukaryotes (Fig. 1), with the $\beta$-1,3-glucan synthases from plants, fungi, Apicomplexa and

21 Stramenopiles consistently emerging as distinct clades. The $\beta$-1,3-glucan synthases of Stramenopiles are further divided into three groups. Clade A, which includes Esi0338_0032, is common to brown algae, diatoms and Oomycetes suggesting that these glycosyltransferases are responsible for the polymerization of the backbones of laminarin, chrysolaminarin and mycolaminarin, respectively. The $\beta$-1,3-glucan synthases of clade $\mathrm{B}$ are only found in 
1 Phytophthora and, therefore, they are likely to be involved in the biosynthesis of the

2 Oomycete cell wall $\beta-1,3$-glucans. Clade $\mathrm{C}$ is unique to brown algae, but the exact role of 3 Esi0033_0138 and Esi0193_0029 is unclear. These $\beta$-1,3-glucan synthases could specifically 4 catalyze the production of laminarin M-series, which is a distinctive feature of brown algae 5 (Read et al., 1996). Alternatively, they might be involved in callose biosynthesis, this 6 molecule having been found in the sieve plates of Laminariales (Parker \& Huber, 1965). In 7 addition, Ectocarpus possesses two proteins (Esi0100_0034 and Esi0243_0020) which are 8 homologous to KRE6, a GH16 family transglycosylase involved in $\beta$-1,6-branching of cell 9 wall $\beta$-1,3-glucans in yeasts (Montijn et al., 1999). Therefore, these two proteins represent 10 good candidates for the synthesis of $\beta-1,6$-linked branches of laminarin. This hypothesis is 11 strengthened by the conservation of KRE6-like proteins in diatoms and Oomycetes. 12 Remarkably, the KRE6-like protein PITG_03335 from P. infestans (Haas et al., 2009) is 13 fused to a GT48 family $\beta$-1,3-glucan synthase. This GT48 module belongs to the clade A 14 (Fig. 4), the very subgroup that we suggest to be responsible for laminarin polymerization. belonging to three different families (GH16: 4 genes; GH17: 1 gene; GH81: 5 genes), and by 17 two exo-1,3-beta-glucanases (family GH5). These numerous laminarinases have homologues 18 in bacteria (family GH16), fungi (families GH5 and GH81) and plants (family GH17), 19 underlining the complexity of laminarin metabolism in brown algae. Laminarin 20 oligosaccharides would be further hydrolyzed by $\beta$-glucosidases of the GH1 (Esi0061_0010, 21 Esi0176_0045 and Esi0212_0019) or GH3 families (Esi0010_0226). The end-product, 22 glucose, would be subsequently phosphorylated by a glucokinase (Esi0000_0270) before 23 entering glycolysis. 


\section{Ectocarpus siliculosus has not retained any trace of the starch metabolism of its red algal}

\section{2 endosymbiont.}

3 The uptake of a red alga by the heterotrophic ancestor of brown algae resulted in the

4 acquisition of photosynthesis. In plants and red algae, the acquisition of photosynthetic 5 capacity following the capture of a cyanobacteria was accompanied by the transformation of 6 the glycogen metabolism of the ancestral host cell into a starch biosynthetic pathway (Ball \& 7 Morell, 2003; Deschamps et al., 2008). In contrast to plants, red algae synthesize starch in their cytoplasm, and not in their plastids, and they use UDP-glucose as the activated sugar instead of ADP-glucose (Nyvall et al., 1999; Viola et al., 2001). The question then arises as to whether there is any remnant of this red algal starch metabolism in extant brown algae.

Starch and glycogen are synthesized and recycled by homologous enzymes. The $\alpha-1,4-$ glucan backbone is produced by starch synthase (GT5) or glycogen synthase (GT3 and GT5), while branching enzymes (GH13) are responsible for the creation of $\alpha-1,6$ branches (Ball \& Morell, 2003). Recycling is mainly performed by the combined action of glycogen/starch phosphorylases (GT35), debranching enzymes (GH13) and $\alpha$-1,4-glucanotransferases (GH77). Additional hydrolytic enzymes are also involved in $\alpha$-1,4-glucan catabolism: $\alpha$ - and $\beta$-amylases (GH13 and GH14) and $\alpha$-glucosidases of the GH31 family (Ball \& Morell, 2003). Starch degradation is specifically initiated by glucan water dikinases (GWDs) and phosphoglucan water dikinases (PWDs), which loosen the crystalline starch granules (Edner et al., 2007). A systematic search for all these enzymes in Ectocarpus did not retrieve any 21 corresponding gene. This seaweed does contain two isoforms of UGP, but these enzymes are not specific for glycogen/starch metabolism as they could also be involved in other biosynthetic pathways (cellulose, laminarin, glycosylation). The ADP-glucose pyrophosphorylase (AGP), which is responsible for ADP-glucose synthesis in plants, is also 


\section{Page 15 of 41}

1 absent from brown algae. Diatom and Oomycete genomes also completely lack starch- or 2 glycogen-related genes. 


\section{Discussion}

\section{The central and storage carbohydrate metabolism in brown algae}

3 Figure 5 shows the central and storage carbon metabolism of E. siliculosus, as

4 reconstructed from the whole genome analyses reported above. In brown algae, this

5 metabolism is profoundly different from what is known for fungi, animals and plants, a

6 uniqueness which is further reinforced in the phylogenomic reconstruction of the metabolic

7 routes for the biosynthesis of cell wall polysaccharides (Michel et al., 2010). With the

8 exception of GDP-mannose pyrophosphorylase (Michel et al., 2010), the enzymes involved in

9 the equilibration of the hexose-phosphate pool and in the generation of activated sugars are

10 conserved with other eukaryotes. However, these key sugars are then used by specific

11 enzymes to generate two unique storage compounds, mannitol and laminarin. Fructose-6-

12 phosphate plays a central role in these pathways. As in the other photosynthetic lineages, this

13 photoassimilate is the precursor of all activated sugars. In brown algae, however, F6P can be

14 also directly transformed into a soluble storage compound (mannitol), without prior

15 conversion into an activated sugar (Fig. 5). Two other activated sugars assume pivotal

16 functions, (i) UDP-glucose (UDPG), as the starting point for the biogenesis of trehalose,

17 laminarin and cellulose, and (ii) GDP-mannose, which is the direct precursor of alginates and

18 the indirect precursor of sulfated fucans (Michel et al., 2010).

This study highlights numerous candidate-genes for which further experimental analyses are needed to confirm their exact biochemical function. Particularly attractive targets are the

21 putative M1Pases and the KRE6-like proteins, which are potentially responsible for the final steps of mannitol and laminarin biosynthesis, respectively. Our findings also open several novel questions as to the nature of the central and storage carbohydrate metabolism in brown

24 algae. To the best of our knowledge, the presence of trehalose was never reported in brown 
1 algae before this study, even in recent reviews (Elbein et al., 2003; Iturriaga et al., 2009). In

2 plants, trehalose and trehalose-6-P (T6P) are in low abundance and difficult to assay. The role

3 of trehalose in green plants is uncertain, but it may regulate starch breakdown (Paul et al.,

4 2008). T6P is considered to be a signal of glucose-6-P (G6P) and UDPG pool size and, hence,

5 to acts as an effective indicator of sucrose. This signal metabolite is thus thought to coordinate

6 carbon metabolism with plant development in response to carbon availability and stress (Paul

7 et al., 2008). By analogy with plants, and since F6P, G6P and UDPG are also interconnected

8 to T6P in Ectocarpus (Fig. 5), trehalose and T6P might act as metabolic regulators in brown

9 algae.

Another unresolved question is the biochemical route which connects mannitol and

11 laminarin and the reason why the majority of the laminarin chains are terminated by a

12 mannitol residue at their reducing end (Read et al., 1996). An attractive working hypothesis is

13 that the addition of a mannitol residue is probably catalyzed by specific glycosyltransferases,

14 for instance the GT48 isoforms which are unique to brown algae (Fig. 4), or by a new,

15 undiscovered family of glycosyltransferases. Considering the catalytic mechanism of

16 glycosyltransferases (Lairson et al., 2008), transfer of activated glucose onto mannitol is

17 likely to be the first step in the biosynthesis of the laminarin M-chains. Indeed, the simplest

18 way to form a covalent bond between mannitol and glucose residues would be for an as yet

19 undescribed GT to use mannitol as an acceptor molecule while the donor sugar substrate

20 would be UDPG. The newly formed glucose-mannitol disaccharide would be subsequently

21 used as an acceptor molecule by a GT48 which would further elongate the laminarin chain.

\section{Origin of central and storage carbohydrates in brown algae}

23 Brown algae and the other Stramenopiles have a rich evolutionary history. This lineage 24 arose from two independent endosymbiotic events, the symbiosis of a protoeukaryote with an 25 alphaproteobacterium and the symbiosis between a protist and a red alga. The 
1 alphaproteobacterial symbiont was progressively converted into mitochondria whereas the

2 red-algal symbiont gave rise to plastids. As red algae had themselves originated from a

3 primary endosymbiosis, between a protist and a cyanobacterium, the emergence of

4 Stramenopiles is described as a secondary endosymbiosis, which involved two eukaryotic

5 cells (Reyes-Prieto et al., 2007). Based on molecular clock analyses calibrated by

6 paleontological constraints, Stramenopiles are thought to have diverged from other major

7 eukaryotic groups about 1 billion years ago and it is also thought that the secondary

8 endosymbiosis happened shortly after the primary endosymbiosis (Douzery et al., 2004).

9 A recent genomic study has further complicated this already complex picture by suggesting

10 that a cryptic endosymbiosis with a green alga, related to extant Prasinophytes such as

11 Micromonas, had occurred in Stramenopiles before the capture of the red algal secondary

12 endosymbiont. This hypothesis is based on the presence, in Stramenopile genomes, of a high

number of genes phylogenetically related to those of green microalgae (Moustafa et al.,

14 2009). However, the only available whole-genome resource for the red algal lineage so far is

15 the genome sequence of $C$. merolae, an unusual red microalga which lacks cell wall, lives in

16 acidic hot water and has a reduced genome (Matsuzaki et al., 2004). Therefore, the

17 interpretation of Moustafa and coworkers is somewhat weakened from the lack of genomic

18 data from archetypal red algae, which may also possess many of the proposed "green algal"

19 genes (Dagan \& Martin, 2009). In this intricate context we discuss below the possible origins

20 of the various carbohydrates from brown algae.

\section{Trehalose was acquired from the red algal endosymbiont}

22 Sucrose is mainly limited to plants, green algae and cyanobacteria, while trehalose is found

23 in a large range of bacteria and Eukaryotes (Salerno \& Curatti, 2003). The widespread

24 occurrence of trehalose led to the hypothesis that this non-reducing disaccharide is a more

25 ancient metabolite than sucrose (Goddijn \& van Dun, 1999). Sucrose metabolism in green 
1 algae and land plants (Chloroplastida) originated from the cyanobiont (Salerno \& Curatti,

2 2003). Consequently, red algae probably acquired sucrose metabolism during primary

3 endosymbiosis as well, even though these photosynthetic organisms are not currently known

4 to produce sucrose (Kremer, 1980). Inspection of the genome of C. merolae (Matsuzaki et al., 5 2004) indicates that this red alga does not contain any sucrose-related gene. Similarly, genes

6 involved in sucrose metabolism are completely absent from Stramenopiles genomes, as

7 deduced from the lack of sucrose synthase, sucrose-phosphate synthase and of invertase.

8 Therefore, it is very likely that the red alga that gave birth to the phaeoplasts of extant

9 Stramenopiles (Reyes-Prieto et al., 2007) had already lost the cyanobacterial sucrose-

10 metabolism when the secondary endosymbiosis took place. Nevertheless, this hypothesis

11 needs additional genomic data on red algae to be definitively confirmed.

12 In the case of trehalose, the question also arises as to the origin of this carbon storage disaccharide in brown algae; whether it was originated from the host or from the red algal

14 endosymbiont. Trehalose synthesis requires two enzyme activities: trehalose-phosphate synthase (TPS) and trehalose-phosphate phosphatase (TPP). Here we show that the six TPS/TPP of Ectocarpus are closely related to their orthologs from C. merolae (Fig. 3). Therefore, it is likely that trehalose metabolism was transmitted into Stramenopiles by the endosymbiont and that the red algal genes supplanted preexisting host orthologs, if any existed. The assimilation of the red algal pathway by the host was probably facilitated by the fact that this pathway already consisted of a single bifunctional enzyme, with both TPP and

21 TPS activities, in ancestral red algae (Fig. 5).

\section{Mannitol: evidence for a major horizontal gene transfer event from Actinobacteria}

Brown algae differ from other eukaryotes, including diatoms and Oomycetes, by their capacity to synthesize an additional carbon storage sugar, mannitol. The conversion of F6P into mannitol involves two enzymatic steps (Fig. 5), catalyzed by M1PDH and M1Pase. 
1 These enzyme activities are indeed rather rare among living organisms and here we show that

2 the M1PDH and putative M1Pase genes from Ectocarpus are closely related to those of Gram

3 positive bacteria (Fig. 2). Based on this close phylogenetic relationship, we propose that, after

4 the divergence of brown algae from diatoms and Oomycetes, these two enzymes were

5 imported into brown algae by a horizontal gene transfer involving an ancestral Gram positive

6 bacterium related to extant Actinobacteria. It is noteworthy that this bacterial phylum is

7 common and diversified in the marine environment (Bull et al., 2005). As reported in more

8 detail in the second part of this study (Michel et al., 2010), this HGT event extended beyond

9 the acquisition of mannitol metabolism. The actinobacterium would also have contributed to

10 the enzyme machinery that synthesizes alginate and hemicelluloses, two major cell wall

11 polysaccharides which are absent from diatoms and Oomycetes. Therefore, this bacterial HGT

12 was probably instrumental in the emergence of complex multicellularity in brown algae, by

13 providing two essential elements in this evolutionary process: (i) extracellular matrix

14 polysaccharides for the construction of multicellular tissues and organs, (ii) and mannitol for

15 the long distance translocation of photoassimilates (Parker \& Huber, 1965).

\section{Micromonas acquired the capacity to synthesize mannitol from brown algae}

In the phylogenetic analyses of M1PDH and putative M1PAses, the Stramenopile genes cluster with their orthologues in Micromonas (Prasinophytes), suggesting that these microalgae feature a mannitol metabolism of brown algal origin. Beyond the robustness and the congruence of the phylogenetic trees (Fig. 2), several pieces of evidence support the direction of this potential HGT, from brown algae to these Prasinophytes: (i), M1PDH and putative M1Pases are standalone genes in Ectocarpus and the closest orthologs of brown algal M1Pases in Micromonas are also standalone proteins, whereas the M1Pases fused to M1PDH are more divergent (Fig. 2b). This suggests that a gene duplication specifically occurred in Micromonas followed by a gene-fusion event, between M1PDH and the putative M1Pase 
1 domains; (ii), M1PDH and putative M1Pase genes are not conserved in other Prasinophyte

2 genomes and are absent from red algae, Chlorophytes, mosses and higher plants. In contrast,

3 orthologues of these genes are present in brown algal EST libraries (Roeder et al., 2005;

4 Wong et al., 2007; Pearson et al., 2009); and (iii), the other genes of actinobacterial origin in

5 Ectocarpus are not present in Prasinophytes (Michel et al., 2010), suggesting that there was

6 not a direct HGT from Actinobacteria to Prasinophytes. Altogether these findings indicate

7 that the mannitol-related genes in Micromonas were acquired relatively recently from a brown

$8 \quad$ alga by these Prasinophytes.

\section{Laminarin biosynthesis is ancestral in Stramenopiles}

The $\beta$-1,3-glucan synthases from brown algae and other Stramenopiles belong to the GT48

11 family, a family that is conserved in most eukaryotic phyla, but which is absent from Bacteria

12 and Archaea. The phylogenetic tree of the GT48 family is congruent with the currently

13 accepted phylogeny of Eukaryotes (Fig. 4). This finding indicates that $\beta$-1,3-glucans were

14 present in the last common ancestor of eukaryotes and that laminarin is an ancestral

15 metabolite in Stramenopiles. The conservation between fungi (Unikonts) and Stramenopiles (Bikonts) of KRE6-like proteins (GH16) and some laminarinases (GH5 and GH81) is also consistent with the ancestral character of laminarin metabolism. It is likely that intracellular $\beta$-1,3-glucans were the ancestral forms of these carbohydrates and that they subsequently evolved to be secreted as extracellular polysaccharides as complex multicellularity emerged. Interestingly, such an evolution occurred independently in fungi and in plants, as well as

21 within the Stramenopiles, with Oomycetes and Laminariales producing both storage and cell wall $\beta$-1,3-glucans (Parker \& Huber, 1965; Bartnicki-Garcia, 1968). 


\section{Insights into the origin and evolution of storage polysaccharides in Eukaryotes}

Biosynthesis and remobilization of carbon stores is a fundamental process in all living cells. Extant Eukaryotes exhibit a striking diversity in the nature of their storage polysaccharides, which probably reflects their complex evolutionary history. We discuss below various evolutionary scenarios which would account for such a high diversity.

\section{The starch metabolism from the red algal endosymbiont was not retained in}

\section{Stramenopiles}

Starch metabolism is a complex process, involving a minimal set of ten different enzymes in Rhodophyceae (Deschamps et al., 2008). From the seminal work of Steven Ball and colleagues on the origin of starch metabolism, it is now well recognized that this biosynthetic pathway, characteristic of Archaeplastida, results from the merging of the glycogen metabolism of the eukaryotic host cell with the starch metabolism of the cyanobacterial endosymbiont (Ball \& Morell, 2003; Deschamps et al., 2008; Plancke et al., 2008). In particular, all the genes required for the complete pathway of glycogen metabolism in heterotrophic Eukaryotes are maintained in red algae. The transition from glycogen to starch metabolism was due to the transfer of a small number of cyanobacterial genes, namely isoamylase (GH13), granule bound starch synthase I (GBSSI, GT5) and $\alpha-(1,4)$ glucanotransferase (GH77), to the host nuclear genome (Deschamps et al., 2008). This fate contrasts with the usual loss of storage polysaccharides by bacterial parasites and obligatory symbionts (Henrissat et al., 2002; Gil et al., 2004). The success of the acquisition of starch metabolism in the Archaeplastida following plastid primary endosymbiosis was probably due to compatibility between biosynthetic pathways in the eukaryotic host cell and in its cyanobiont. The limited number of transferred genes needed for this metabolic transformation was also a favorable factor (Deschamps et al., 2008). 
In contrast to Unikonts and Archaeplastida, the genomes of brown algae, diatoms and

2 Oomycetes completely lack starch- or glycogen-related genes. It follows that the starch

3 metabolism of the red algal secondary endosymbiont was not retained in Stramenopiles.

4 Instead, their storage compounds consist of laminarin, a $\beta$-1,3-glucan. Here we demonstrate

5 that the metabolism of $\beta$-1,3-glucans involves ancient eukaryotic enzymes. Therefore, we

6 propose that the carbon storage machinery of the protistean ancestor of Stramenopiles was

7 exclusively dedicated to the synthesis of $\beta$-1,3-glucans, a metabolism totally unrelated to the

8 biosynthesis of $\alpha-1,4$-glucans in its red algal endosymbiont. Based on the reasoning

9 developed by Ball and coworkers for Archaeplastida, and as observed for obligatory

10 symbionts (Henrissat et al., 2002; Gil et al., 2004), this metabolic incompatibility resulted in a

11 rapid loss of the starch metabolism of the engulfed red alga.

Synapomorphies of Stramenopiles with respect to carbon storage metabolism do not support the Chromalveolate hypothesis

In comparison to the other major eukaryotic groups, Stramenopiles and Alveolates are rather closely related lineages (Baldauf, 2008). They are thought to constitute the kingdom of

16 Chromoalveolates, distinct from the two other monophyletic super-groups, Unikonts and 17 Archaeplastida (Fig. 1). In spite of their phylogenetic relatedness with Stramenopiles, Alveolates such as Dinoflagellates and Apicomplexa accumulate starch in their cytoplasm (Coppin et al., 2005). As observed in the Archaeplastida, the starch pathway of Alveolates is a hybrid metabolism. The indirect debranching enzymes from Apicomplexa have homologues

21 in animals and fungi, but not in Archaeplastida, indicating that the aveolate host cell 22 contained its own pathway for glycogen synthesis (Coppin et al., 2005). Phylogenetic analyses of other key enzymes for starch synthesis demonstrate that, following the secondary endosymbiosis, the ancestral host-cell glycogen pathway evolved into a genuine starch metabolism through the transfer of red algal starch-related genes (Coppin et al., 2005). 
Altogether, Stramenopiles and Alveolates have evolved completely different pathways for carbon storage: (i), the host cells of Stramenopiles and Alveolates differed in their ancestral carbon storage polysaccharide forms, $\beta$-1,3-glucan versus glycogen respectively; (ii), these metabolic divergences resulted in radically different fates for red algal endosymbionts, complete loss of the red algal starch pathway in Stramenopiles as opposed to transition from glycogen to red algal-like starch metabolism in Alveolates. It is very unlikely that two lineages with the same original genetic background, that is, genomes from the same ancestral host and rhodobiont cells would have evolved two so strikingly different carbon storage metabolisms. It follows that extant Stramenopiles and Alveolates arose from related, yet distinct eukaryotic host cells and from independent secondary endosymbiotic events. The occurrence of two independent, secondary endosymbiotic events contradicts the Chromalveolate hypothesis (Cavalier-Smith, 1999).

\section{Origin of carbon storage polysaccharides in Eukaryotes}

In conclusion, compared to the other eukaryotic phyla, Stramenopiles feature very distinctive traits in their storage carbon metabolism. Long-term carbon storage is based on laminarin, a soluble vacuolar $\beta$-1,3-glucan, whereas most other eukaryotes use polysaccharides based on $\alpha-1,4$-glucan, either on a soluble (glycogen) or insoluble (starch) form. Interestingly, Euglenophytes (Excavata) and Haptophytes, two lineages independent from Stramenopiles (Fig. 1), also use $\beta$-1,3-glucans as carbon storage (Kiss et al., 1988; Andersen, 2004).

The question then arises of the origin of these two classes of storage polysaccharides and whether one class predates the other. As shown in this study, the polymerization of $\beta-1,3-$ glucans is catalyzed by GT48, a protein family conserved in most Eukaryotic groups, but absent in Prokaryotes. Therefore, we propose that $\beta$-1,3-glucans are the archetypal storage polysaccharide in Eukaryotes (Fig. 6). In contrast, most of the genes involved in eukaryotic 
1 glycogen metabolism are shared with bacteria (Ball \& Morell, 2003), suggesting that 2 glycogen is of bacterial origin. It is difficult to trace back the bacterial group which could 3 have transmitted this metabolism to Eukaryotes. The most parsimonious scenario is that 4 Eukaryotes acquired glycogen metabolism from the alphaproteobacterial progenitor of 5 mitochondria (Fig. 6). If this is true, two forms of carbon storage, $\beta$-1,3-glucan and glycogen, 6 may have been transiently present after the mitochondrial endosymbiosis in all of the 7 emerging eukaryote lineages. In most of them, glycogen then supplanted $\beta-1,3$ glucan as the 8 form of carbon storage. However, the enzymes responsible for $\beta$-1,3-glucan synthesis were 9 conserved in several eukaryotic groups (e.g. fungi, plants, Oomycetes, brown algae), where they evolved to produce extracellular $\beta$-1,3-glucans. In Archaeplastida and Alveolates, the 11 ancestral glycogen metabolism of the host cell evolved into a starch metabolism, after the 12 plastid primary and secondary endosymbioses, respectively (Coppin et al., 2005; Deschamps 13 et al., 2008).

14 In the ancestors of extant Stramenopiles, the bacterial glycogen metabolism was lost (Fig. 15 6). As discussed above, we propose that this evolutionary route was probably completed 16 before the secondary endosymbiosis took place, thus preventing the incorporation of the red 17 algal starch metabolism into the host cell. Finally, in brown algae only, a significant HGT happened with an actinobacterium, leading to the de novo acquisition of an additional pathway for carbon storage, based on mannitol. Considering also that they have inherited an ancestral trehalose metabolism from their red algal endosymbiont, brown algae have evolved 21 a very unique central and storage carbon metabolism. From a similar phylogenomic analysis 22 of the enzymes involved in cell wall polysaccharides synthesis, we show that a variety of other major speciation events further contributed to the striking metabolic uniqueness of this lineage (Michel et al., 2010). 


\section{$1 \quad$ References}

Andersen RA. 2004. Biology and systematics of Heterokont and Haptophyte algae. American Journal of Botany 91: 1508-1522.

Armbrust EV, et al. 2004. The genome of the diatom Thalassiosira pseudonana: ecology, evolution, and metabolism. Science 306: 79-86.

Assali NE, Martin WF, Sommerville CC, Loiseaux-de Goer S. 1991. Evolution of the Rubisco operon from prokaryotes to algae: structure and analysis of the rbcS gene of the brown alga Pylaiella littoralis. Plant Molecular Biology 17: 853-863.

Baldauf SL. 2008. An overview of the phylogeny and diversity of eukaryotes. Journal of Systematics and Evolution 46: 263-273.

Ball SG, Morell MK. 2003. From bacterial glycogen to starch: understanding the biogenesis of the plant starch granule. Annual Review of Plant Biology 54: 207-233.

Bartnicki-Garcia S. 1968. Cell wall chemistry, morphogenesis, and taxonomy of fungi. Annual Review of Microbiology 22: 87-108.

Bateman A, et al. 2004. The Pfam protein families database. Nucleic Acids Research 32: $138-141$.

Beattie A, Hirst EL, Percival E. 1961. Studies on the metabolism of the Chrysophyceae. Comparative structural investigations on leucosin (chrysolaminarin) separated from diatoms and laminarin from the brown algae. Biochemical Journal 79: 531-537.

Ben Ali A, De Baere R, Van der Auwera G, De Wachter R, Van de Peer Y. 2001. Phylogenetic relationships among algae based on complete large-subunit rRNA sequences. International Journal of Systematic and Evolutionary Microbiology 51: 737-749.

Bodyl A, Stiller JW, Mackiewicz P. 2009. Chromalveolate plastids: direct descent or multiple endosymbioses? Trends in Ecology and Evolution 24: 119-121. 
1 Bowler C, et al. 2008. The Phaeodactylum genome reveals the evolutionary history of diatom 2 genomes. Nature 456: 239-244.

3 Bull AT, Stach JE, Ward AC, Goodfellow M. 2005. Marine actinobacteria: perspectives, challenges, future directions. Antonie Van Leeuwenhoek 87: 65-79.

Cantarel BL, Coutinho PM, Rancurel C, Bernard T, Lombard V, Henrissat B. 2009. The Carbohydrate-Active EnZymes database (CAZy): an expert resource for Glycogenomics. Nucleic Acids Research 37: D233-238.

Cardenas ML, Cornish-Bowden A, Ureta T. 1998. Evolution and regulatory role of the hexokinases. Biochimica Biophysica Acta 1401: 242-264.

Cavalier-Smith T. 1999. Principles of protein and lipid targeting in secondary symbiogenesis: Euglenoid, Dinoflagellate, and Sporozoan plastid origins and the Eukaryote family tree. The Journal of Eukaryotic Microbiology 46: 347-366.

Charrier B, et al. 2008. Development and physiology of the brown alga Ectocarpus siliculosus: two centuries of research. New Phytologist 177: 319-332.

Cock JM, et al. 2010. The Ectocarpus genome and the independent evolution of multicellularity in the brown algae. Nature In the press.

Coppin A, Varre JS, Lienard L, Dauvillee D, Guerardel Y, Soyer-Gobillard MO, Buleon A, Ball S, Tomavo S. 2005. Evolution of plant-like crystalline storage polysaccharide in the protozoan parasite Toxoplasma gondii argues for a red alga ancestry. Journal of Molecular Evolution 60: 257-267.

Dagan T, Martin W. 2009. Microbiology. Seeing green and red in diatom genomes. Science 324: 1651-1652.

Derelle E, et al. 2006. Genome analysis of the smallest free-living eukaryote Ostreococcus tauri unveils many unique features. Proceedings of the National Academy of Sciences of the United States of America 103: 11647-11652. 
1 Deschamps P, et al. 2008. Metabolic symbiosis and the birth of the plant kingdom. Molecular Biology and Evolution 25: 536-548.

Douzery EJ, Snell EA, Bapteste E, Delsuc F, Philippe H. 2004. The timing of eukaryotic evolution: does a relaxed molecular clock reconcile proteins and fossils? Proceedings of the National Academy of Sciences of the United States of America 101: 15386-15391.

Edner C, et al. 2007. Glucan, water dikinase activity stimulates breakdown of starch granules by plastidial beta-amylases. Plant Physiology 145: 17-28.

Elbein AD, Pan YT, Pastuszak I, Carroll D. 2003. New insights on trehalose: a multifunctional molecule. Glycobiology 13: 17R-27R.

Gil R, Latorre A, Moya A. 2004. Bacterial endosymbionts of insects: insights from comparative genomics. Environmental Microbiology 6: 1109-1122.

Goddijn OJ, van Dun K. 1999. Trehalose metabolism in plants. Trends in Plant Science 4: $315-319$

Gravot A, Dittami SM, Rousvoal S, Heyl K, Eggert A, Cock JM, Boyen C, Boucherau A, Tonon T. 2010. Diurnal oscillations of metabolite abundances in Ectocarpus siliculosus and genome analysis provide new insights into central metabolic processes of brown algae. Submitted.

Gschloessl B, Guermeur Y, Cock JM. 2008. HECTAR: a method to predict subcellular targeting in heterokonts. BMC Bioinformatics 9: 393.

Guindon S, Gascuel O. 2003. A simple, fast, and accurate algorithm to estimate large phylogenies by maximum likelihood. Systematic Biology 52: 696-704.

Haas BJ, et al. 2009. Genome sequence and analysis of the Irish potato famine pathogen Phytophthora infestans. Nature 461: 393-398.

Henrissat B. 1991. A classification of glycosyl hydrolases based on amino acid sequence similarities. Biochemical Journal 280: 309-316. 
1 Henrissat B, Coutinho PM, Davies GJ. 2001. A census of carbohydrate-active enzymes in

2 the genome of Arabidopsis thaliana. Plant Molecular Biology 47: 55-72.

3 Henrissat B, Deleury E, Coutinho PM. 2002. Glycogen metabolism loss: a common marker of parasitic behaviour in bacteria? Trends in Genetics 18: 437-440.

Ikawa T, Watanabe T, Nisizawa K. 1972. Enzymes involved in the last steps of the biosynthesis of mannitol in brown algae. Plant and Cell Physiology 13: 1017-1029.

Iturriaga G, Suarez R, Nova-Franco B. 2009. Trehalose metabolism: from osmoprotection to signaling. International Journal of Molecular Sciences 10: 3793-3810.

Iwamoto K, Shiraiwa Y. 2005. Salt-regulated mannitol metabolism in algae. Marine Biotechnology 7: 407-415.

Katoh K, Misawa K, Kuma K, Miyata T. 2002. MAFFT: a novel method for rapid multiple sequence alignment based on fast Fourier transform. Nucleic Acids Research 30: 30593066.

Keeling PJ. 2009. Chromalveolates and the evolution of plastids by secondary endosymbiosis. The Journal of Eukaryotic Microbiology 56: 1-8.

Kiss JZ, Roberts EM, Brown RM, Triemer RE. 1988. X-ray and dissolution studies of paramylon storage granules from Euglena. Protoplasma 146: 150-156.

Kremer BP. 1980. Taxonomic implications of algal photoassimilate patterns. European Journal of Phycology 15: 399-409.

Krogh A, Larsson B, von Heijne G, Sonnhammer EL. 2001. Predicting transmembrane protein topology with a hidden Markov model: application to complete genomes. Journal of Molecular Biology 305: 567-580.

Kumar S, Tamura K, Nei M. 2004. MEGA3: Integrated software for Molecular Evolutionary Genetics Analysis and sequence alignment. Briefings in Bioinformatics $\mathbf{5}$ : 150-163. 
1 Lairson LL, Henrissat B, Davies GJ, Withers SG. 2008. Glycosyltransferases: structures,

2 functions, and mechanisms. Annual Review of Biochemistry 77: 521-555.

3 Lane CE, Archibald JM. 2009. Reply to Body, Stiller and Mackiewicz: “'Chromalveolate plastids: direct descent or multiple endosymbioses?', Trends in Ecology and Evolution 24: 121-122.

Liaud MF, Lichtle C, Apt K, Martin W, Cerff R. 2000. Compartment-specific isoforms of TPI and GAPDH are imported into diatom mitochondria as a fusion protein: evidence in and Evolution 17: 213-223.

\section{Liberator P, Anderson J, Feiglin M, Sardana M, Griffin P, Schmatz D, Myers RW.} 1998. Molecular cloning and functional expression of mannitol-1-phosphatase from the

Lobban CS, Harrison PJ. 1994. Seaweed Ecology and Physiology, 2nd ed. Cambridge University Press, New York, USA.

Matsuzaki M, et al. 2004. Genome sequence of the ultrasmall unicellular red alga Cyanidioschyzon merolae 10D. Nature 428: 653-657.

Merchant SS, et al. 2007. The Chlamydomonas genome reveals the evolution of key animal and plant functions. Science 318: 245-250.

Michel G, Tonon T, Scornet D, Cock JM, Kloareg B. 2010. The carbohydrate metabolism of the brown alga Ectocarpus siliculosus.

Part II: novel insights into the evolution of cell wall polysaccharides. New Phytologist In preparation.

Montijn RC, Vink E, Muller WH, Verkleij AJ, Van Den Ende H, Henrissat B, Klis FM. 1999. Localization of synthesis of beta1,6-glucan in Saccharomyces cerevisiae. Journal of Bacteriology 181: 7414-7420. 
1 Moulin P, Crépineau F, Kloareg B, Boyen C. 1999. Isolation and characterization of six 2 cDNAs involved in carbon metabolism in Laminaria digitata (Phaeophyceae). Journal of $3 \quad$ Phycology 35: 1237-1245.

Moustafa A, Beszteri B, Maier UG, Bowler C, Valentin K, Bhattacharya D. 2009. Genomic footprints of a cryptic plastid endosymbiosis in diatoms. Science 324: 1724-1726.

Nyvall P, Pelloux J, Davies HV, Pedersen M, Viola R. 1999. Purification and characterisation of a novel starch synthase selective for uridine 5'-diphosphate glucose from the red alga Gracilaria tenuistipitata. Planta 209: 143-152.

Parker BC, Huber J. 1965. Translocation in Macrocystis. II. Fine structure of the sieve tubes. Journal of Phycology 1: 172-179.

Paul MJ, Primavesi LF, Jhurreea D, Zhang Y. 2008. Trehalose metabolism and signaling. Annual Review of Plant Biology 59: 417-441.

Pearson GA, et al. 2009. An Expressed Sequence Tag Analysis of the Intertidal Brown Seaweeds Fucus serratus (L.) and F. vesiculosus (L.) (Heterokontophyta, Phaeophyceae) in Response to Abiotic Stressors. Marine Biotechnology In the press.

Percival EGV, Ross AG. 1951. The constitution of laminarin. Part II. The soluble laminarin of Laminaria digitata. Journal of the Chemical Society: 720 - 726.

Plancke C, et al. 2008. Pathway of cytosolic starch synthesis in the model glaucophyte Cyanophora paradoxa. Eukaryotic Cell 7: 247-257.

Read SM, Currie G, Bacic A. 1996. Analysis of the structural heterogeneity of laminarin by electrospray-ionisation-mass spectrometry. Carbohydrate Research 281: 187-201.

Reyes-Prieto A, Weber AP, Bhattacharya D. 2007. The origin and establishment of the plastid in algae and plants. Annual Review of Genetics 41: 147-168. 
1 Richter DFE, Kirst GO. 1987. D-mannitol dehydrogenase and D-mannitol-1-phosphate

2 dehydrogenase in Platymonas subcordiformis: some characteristics and their role in osmotic adaptation. Planta 170: 528-534.

Ridder IS, Dijkstra BW. 1999. Identification of the $\mathrm{Mg}^{2+}$-binding site in the P-type ATPase and phosphatase members of the HAD (haloacid dehalogenase) superfamily by structural similarity to the response regulator protein CheY. Biochemical Journal 339: 223-226.

Roeder V, Collén J, Rousvoal S, Corre E, Leblanc C, Boyen C. 2005. Identification of stress gene transcripts in Laminaria digitata (Phaeophyceae) protoplast cultures by expressed sequenced tag analysis. Journal of Phycology 41: 1227-1235.

Salerno GL, Curatti L. 2003. Origin of sucrose metabolism in higher plants: when, how and why? Trends in Plant Science 8: 63-69.

Schmitz K, Lobban CS. 1976. A survey of translocation in Laminariales (Phaeophyceae). Marine Biology 36: 207-216.

Tyler BM, et al. 2006. Phytophthora genome sequences uncover evolutionary origins and mechanisms of pathogenesis. Science 313: 1261-1266.

Viola R, Nyvall P, Pedersen M. 2001. The unique features of starch metabolism in red algae. Proceedings of the Royal Society B-Biological Sciences 268: 1417-1422.

Wang MC, Bartnicki-Garcia S. 1974. Mycolaminarans: storage (1,3)- $\beta$-D-glucans from the cytoplasm of the fungus Phytophthora palmivora. Carbohydrate Research 37: 331-338.

Wong TKM, Ho CL, Lee WW, Rahim RA, Phang SM. 2007. Analyses of expressed sequence tags from Sargassum binderi (Phaeophyta). Journal of Phycology 43: 528-534.

Worden AZ, et al. 2009. Green evolution and dynamic adaptations revealed by genomes of the marine picoeukaryotes Micromonas. Science 324: 268-272. 


\section{Page 33 of 41}

1 Yamaguchi T, Ikawa T, Nisizawa K. 1966. Incorporation of radioactive carbon from

$2 \mathrm{H}^{14} \mathrm{CO}_{3}^{-}$into sugar constituents by a brown alga, Eisenia bicyclis, during photosynthesis

3 and its fate in the dark. Plant and Cell Physiology 7: 217-229.

4 


\section{$1 \quad$ Figure legends}

2 Fig. 1. Simplified phylogeny of the major groups of eukaryotes (adapted from Baldauf, 3 2008).

Fig. 2. Unrooted phylogenetic tree of the mannitol-1-phosphate 5-dehydrogenases (A) and of the putative mannitol-1-phosphatases (B). The phylogenetic trees were derived using the Maximum Likelihood (ML) approach with the program PhyML (Guindon \& Gascuel, 2003). Numbers indicate the bootstrap values in the ML analysis. The full listing of the aligned proteins is reported in Supporting Information Table S3. The sequences marked by black diamonds correspond to the Ectocarpus proteins.

Fig. 3. Comparison of phylogenetic trees of modular trehalose-phosphate synthases (A) and of their appended trehalose-phosphate phosphatase modules (B). These phylogenetic trees were derived using the Maximum Likelihood (ML) approach with the program PhyML (Guindon \& Gascuel, 2003). Each tree has been rooted using the bacterial orthologs. Numbers indicate the bootstrap values in the ML analysis. The full listing of the aligned proteins is reported in Supporting Information Table S3. The sequences marked by black diamonds and a square correspond to the proteins from the brown alga Ectocarpus siliculosus ( $\bullet$ ) and the red alga Cyanidioschyzon merolae (๘), respectively.

Fig. 4. Unrooted phylogenetic tree of the $\beta$-1,3-glucan synthases (family GT48). This phylogenetic tree was derived using the Maximum Likelihood (ML) approach with the program PhyML (Guindon \& Gascuel, 2003). Numbers indicate the bootstrap values in the ML analysis. The full listing of the aligned proteins is reported in Supporting Information Table S3. The sequences marked by black diamonds correspond to the Ectocarpus proteins.

Fig. 5. Schematic representation of the central and storage carbon metabolism of Ectocarpus siliculosus based on genome annotation data. Gene products contributing to 
1 these pathways are indicated by the code XXXX_YYYY where XXXX indicates the

2 superconting number and YYYY the gene number of the locus on this supercontig. The 3 prefix Esi has been omitted to improve clarity. Mannitol metabolism: M1PDH, mannitol-1-

4 phosphate 5-dehydrogenase; M1Pase, mannitol-1-phosphatase; M2DH, mannitol-25 dehydrogenase; FK, fructokinase. Laminarin synthesis: GPI, glucose-6-phosphate 6 isomerase; PGM, phosphoglucomutase; UGP, UDP-glucose-pyrophosphorylase; GT48, $\beta$ 7 1,3-glucan synthases (family GT48); KRE6-like proteins, putative 1,6- $\beta$-transglucosylases 8 (family GH16). The question mark indicates the absence of an identified candidate for the 9 reaction that links mannitol to $\beta-1,3$-glucan to produce the M-series of laminarin. The 10 dotted arrows and boxes indicate biosynthetic pathways related to cell wall 11 polysaccharides (cellulose, alginates and sulfated fucans).

12 Fig. 6. Schematic flowchart illustrating the evolution of carbon storage polysaccharides 13 within major eukaryotic lineages. Endosymbiosis events are indicated by dotted lines. 14 ME: Mitochondrial endosymbiosis; PE: plastid primary endosymbiosis; SE: plastid 15 secondary endosymbiosis. 


\section{Bikonts}

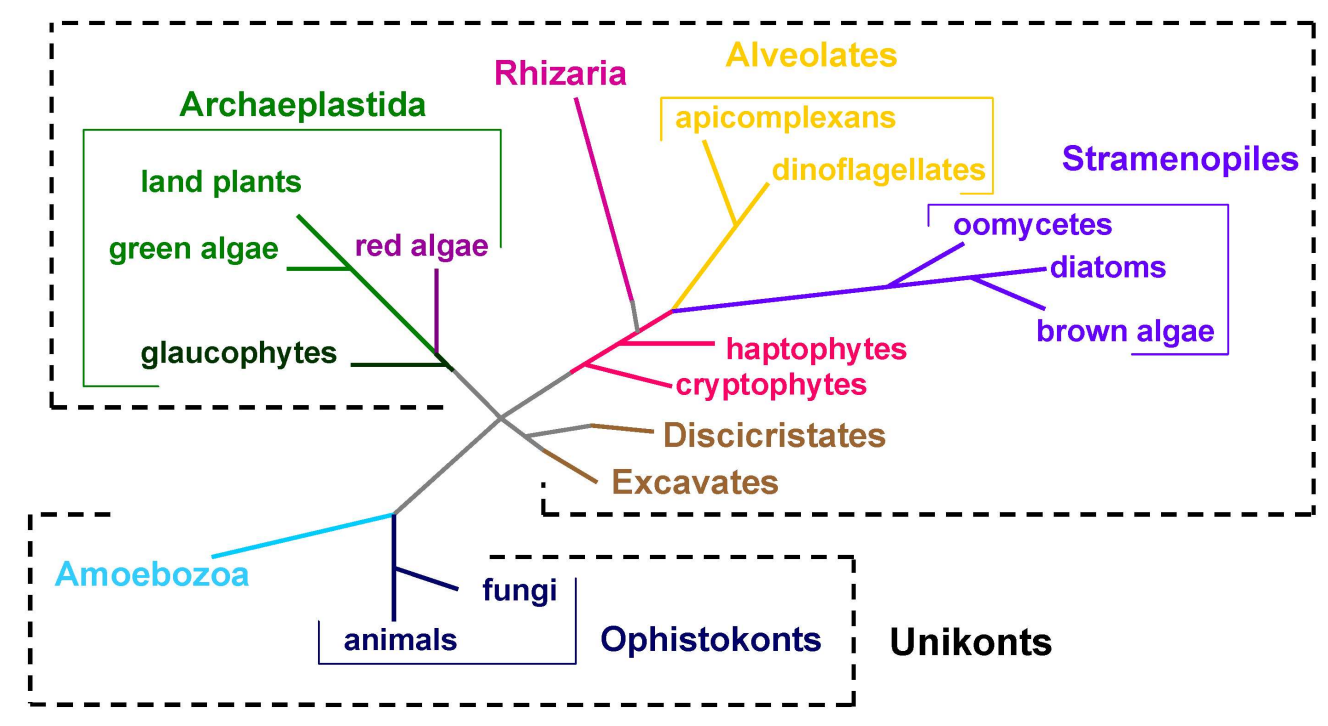

Figure 1

$239 \times 138 \mathrm{~mm}(300 \times 300 \mathrm{DPI})$ 


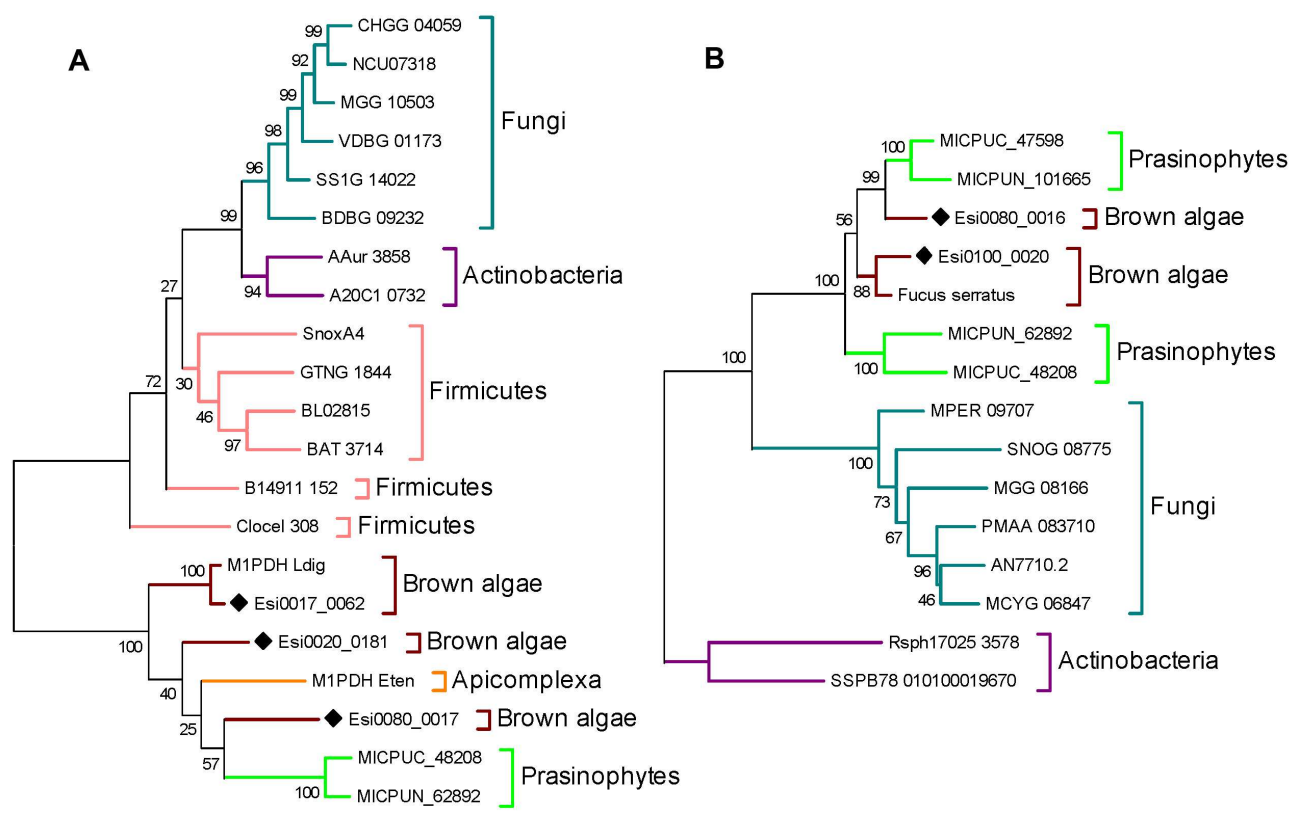

Figure 2

$215 \times 133 \mathrm{~mm}(300 \times 300$ DPI) 

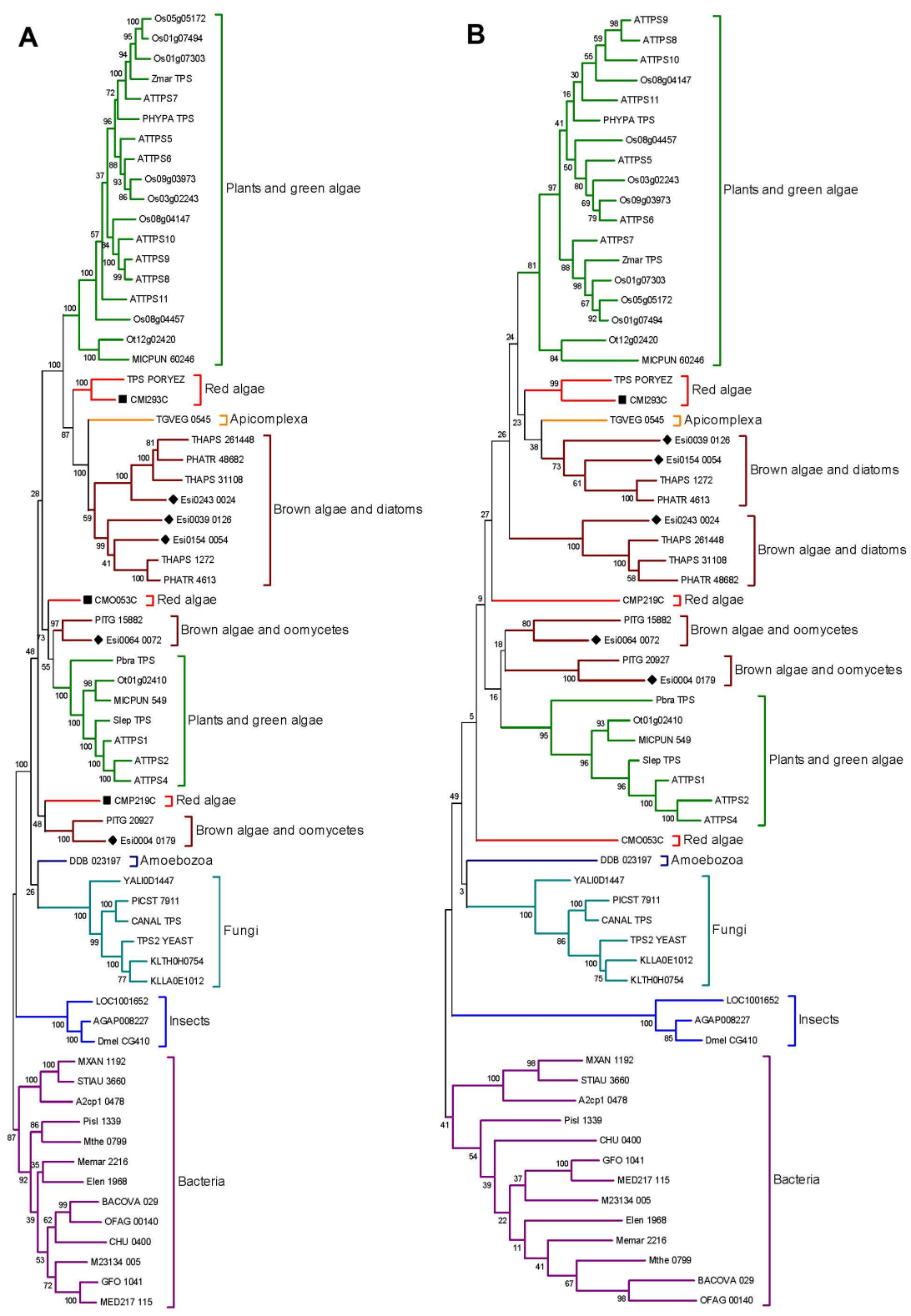

Figure 3

$164 \times 234 \mathrm{~mm}(300 \times 300 \mathrm{DPI})$ 


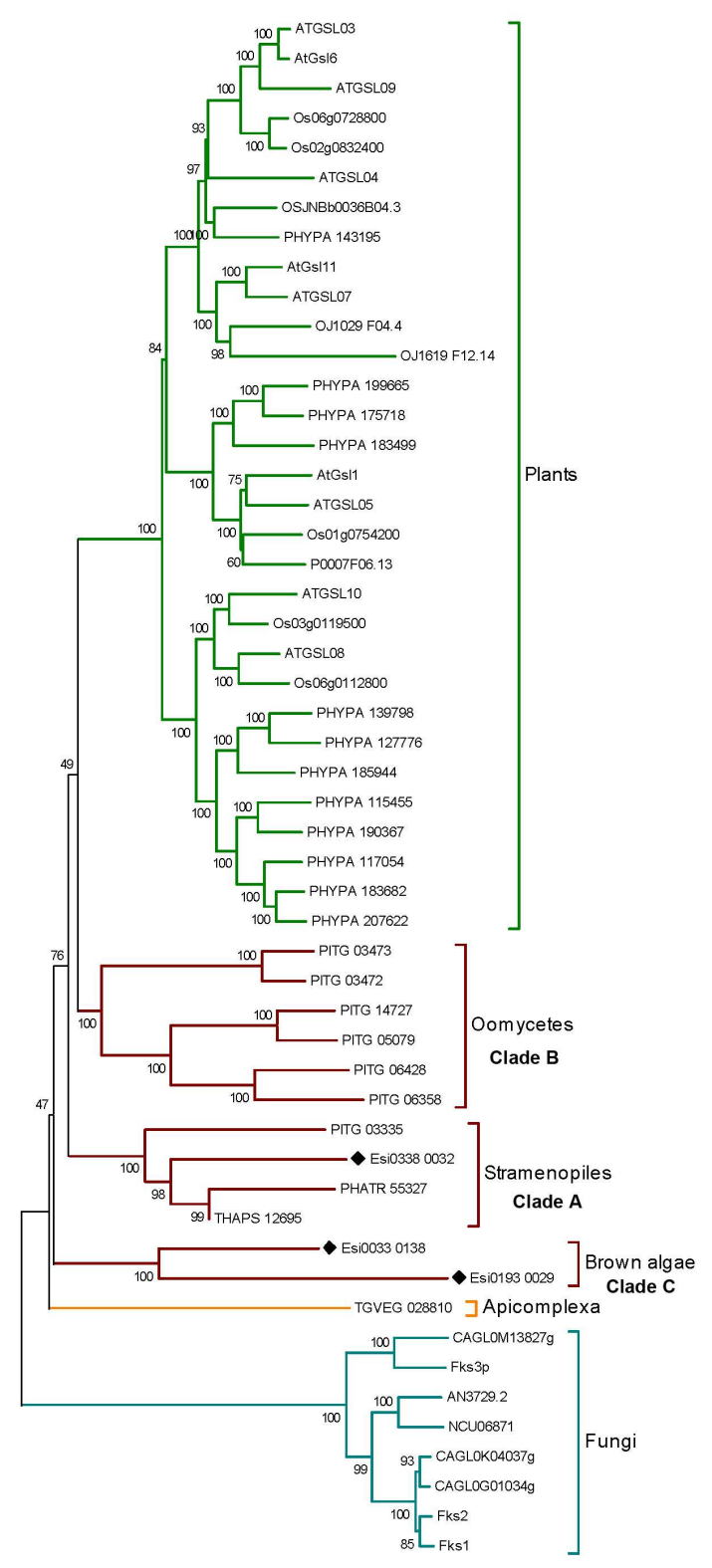

Figure 4

$109 \times 234 \mathrm{~mm}(300 \times 300 \mathrm{DPI})$ 




Figure 5

$173 \times 219 \mathrm{~mm}(300 \times 300 \mathrm{DPI})$ 


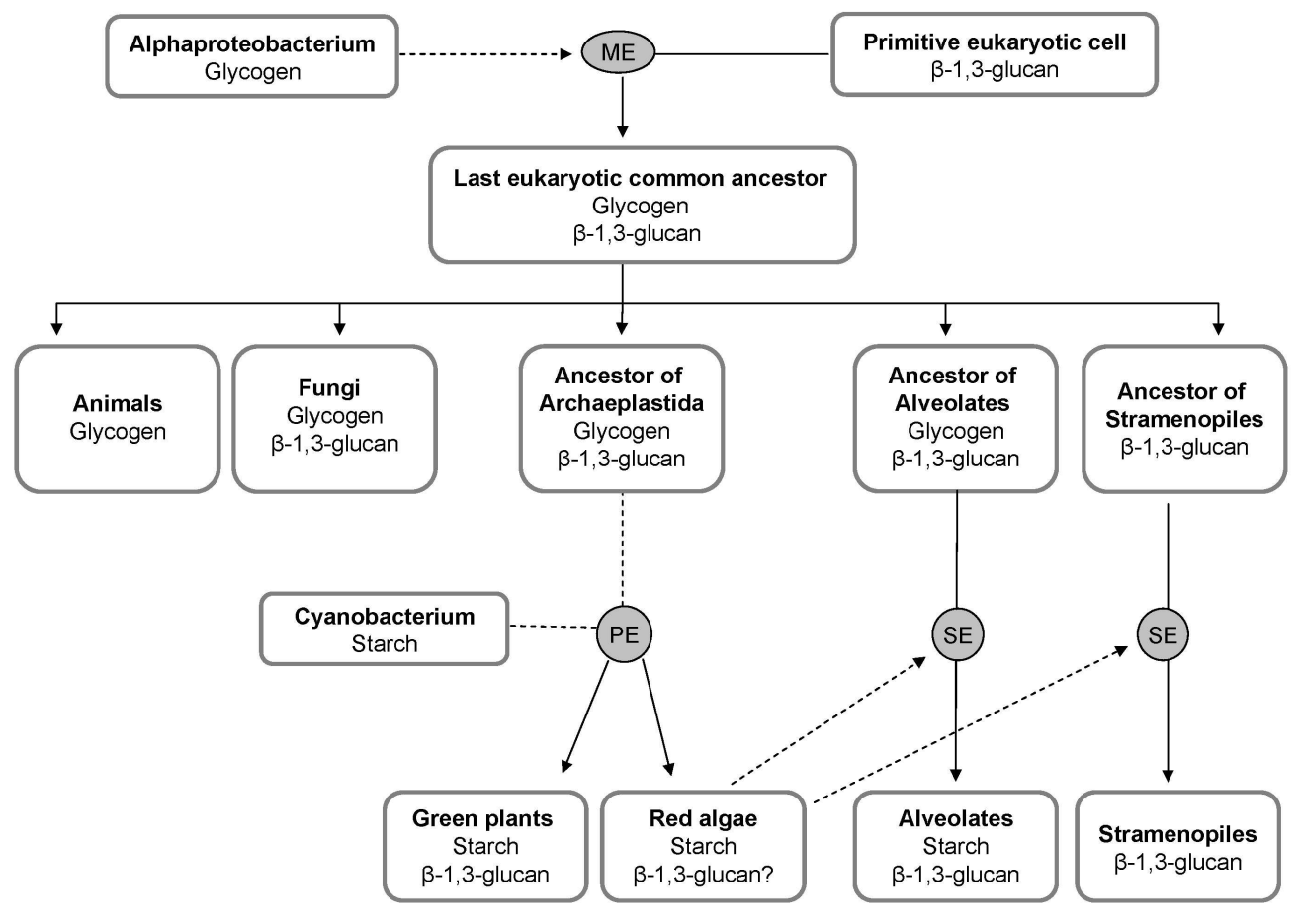

Figure 6

$236 \times 166 \mathrm{~mm}(300 \times 300$ DPI $)$ 